\title{
Performance Measure Analysis between Anisotropic Diffusion Filter and Bilateral Filter for Post Processing of Fractal Compressed Medical Images
}

\author{
S. Padmashree \\ Associate professor, \\ GSSS Institute of Engineering and Technology for \\ women KRS Road, \\ Karnataka, Mysuru, India
}

\author{
Rohini Nagapadma, PhD \\ Professor, \\ The National Institute of Engineering,Karnataka, \\ Mysuru, India.
}

\begin{abstract}
Filtering is Prime important processes in Medical Image processing applications. Any post processing process aims in the removal of unwanted noise which usually corrupts the image quality and perception. This research paper focuses on searching effective De noising filters for post processing of Fractal compressed Images on Medical Images like CT of Bone , MR Images of Brain ,Mammograms, Ultrasound Images of uterus. In this work Fractal Image Compression (FIC) a lossy compression scheme based on contractive mapping theorem is employed to map the Range blocks and Domain blocks by using the property of self similarity in the images. We have used two types of filters namely anisotropic diffusion filter and bilateral filter for the removal of noise in Medical images. The Peak signal to noise ratio (PSNR) was measured after applying the two different filters and a comparative analysis of PSNR values before and after filtering was recorded. The simulated results obtained showed an increase in PSNR value for bilateral filter than with anisotropic filter and also the quality of the image was improved.
\end{abstract}

\section{Keywords}

Fractal Theory, Iterated Function systems, quad-tree, Range blocks, Filtering, Isotropic filters, Bilateral filters, medical imaging, PSNR

\section{INTRODUCTION}

The huge development in the field of digital electronics computer engineering and medical imaging has resulted in generation of large amount of data in the digital form. This progress is being observed in wide field of digital images which covers scanned documents, drawings, images from digital or video cameras, satellite images, medical images, works of computers and many more. Medical imaging has developed a lot in the past years with invention of many new imaging modalities and faster equipments for image acquisition. Hence there is a requirement for long- lasting storage of any data to make it profitable particularly in the medical field. Hospital Information Systems contain a large number of medical examination results. The doctors can familiarize themselves with the case history and make a diagnosis based on many different examination results Unfortunately, the hospital databases are growing rapidly each day tens or hundreds of images being produced and storage and retrieval of these data is becoming a challenging task.
Both the sharing and storage aspects of digital data have their inherent problems which puts limitation to their usage. The increase of throughput used in communication connections has led to the development of data compression techniques. Data compression is the technique to reduce the redundancies in data representation in order to decrease data storage requirements and hence communication costs. Over the years, many different compression methods, algorithms and file formats have been developed. In still images compression, there are many different approaches and each one of them produces many compression methods. However all techniques prove to be useful only in a limited usage area. The image compression methods [1] are also much desired or even more necessary in medical field. However, medical images [2] require special compression techniques [3] as it profoundly influences the quality of diagnosis. Low quality medical image, distortions in the image or untrue details may lead to wrong diagnosis and hence a wrong line of treatment, which can be detrimental to human life. Thus any processing done on such images, including compression, should not interfere with the information carried by the images. Hence the compressed medical images need to be filtered to remove the noise that gets added up during the process of image acquisition. In earlier days any non linear filter like median filter, mean filter [4] was suitable for image enhancement but these filters were not efficient enough to remove the acquired noise in the image. These noises can degrade the image and hence should be filtered with suitable de noising filters. As per the Review conducted there are many de noising filters. available but only certain de nosing filters are suitable for removal of noise in Medical Images like MRI and ultrasound images. The goal of image compression is to remove redundancy present in a data in a way that enables acceptable image reconstruction. There are numerous lossless and lossy compression techniques. Lossless compression techniques allow the image to be compressed by reducing the redundancy in the data where decompressed data is an exact copy of the original with no loss of data. Huffman coding and Lempel Ziv (LZV) are the examples for Lossless compression. However, Lossy compression sacrifices the exact reproduction of the original. Lossy image compressions are useful in applications such as broadcast television, videoconferencing, and facsimile transmission, in which a certain amount of error is an acceptable trade-off for increased compression performance. Methods for lossy compression are Fractal compression, Transform coding, Fourier-related transform, 
DCT (Discrete Cosine Transform) and Wavelet transform. JPEG is an example of Lossy compression. Though JPEG is the currently used most popular compression method, the fractal image compression [5][6][7] is an another lossy compression method which is getting an edge over the JPEG across the globe.

In this paper an attempt is being made to implement Fractal Image compression technique using quad-tree Fractal Image Compression for Medical images. The decompressed image is filtered to remove noise using anisotropic filters and Bilateral filters. The main objective of the paper is to identify the best de noising filter to de noise the decompressed image obtained through FIC for medical Images like Mammograms and Magnetic Resonance image of Brain, Ultrasound images of uterus and Commuted tomography (CT)of Bone each size $256 \times 256$ and also to evaluate PSNR values after the application of filters.

Section II deals with the literature survey conducted in the current research work. The Fundamentals of Fractal image compression is discussed in section III and section IV deals de noising filters. Proposed de noising block diagram for Fractal Image compression technique with de noising filter is discussed in section V. Parameters to be evaluated is discussed in section VI. Results and Discussions of the proposed work are being discussed in section VII .conclusions of the current work is being mentioned in section VIII.

\section{RELATED WORK}

Image compression deals with the removal of redundant data preserving the quality of the image. Fractal Image Compression is one such effective lossy compression technique. Fractal theory was first developed by Benoit. B. Mandelbrot, a mathematician in Mathematical geometry. Later M Barnsley introduced the fundamental principle of fractal image compression in the year 1988 [8]. Fractal image compression is also called fractal image encoding as the compressed image is represented by contractive transforms called Affine transforms and mathematical functions for reconstruction of the image [9]. Affine transforms ensures that the distance between any two points on the transformed image to be less than the distance between the same points on the original image. These transforms are composed by the union of number of affine mappings on the entire image known as the Iterated Function Systems (IFS) [10-12]. The fractal image compression based on IFS was complicated and was still not practical. Arnaud Jacquin settled the problem with the partition of IFS in 1990.Jacquin partitioned the image into sub images called the Range Blocks and PIFS were applied on the sub images rather than the whole image. In any Fractal compression system the first decision is to choose the type of image partition for the range blocks formation. A wide variety of partitions have been investigated. Fixed size square blocks are the simplest possible partition They are easy to implement but its performance decreases for images The solution for this problem is to use some adaptive scheme for block size so that large blocks are assigned for low detail region and small blocks for significant detail region Two approaches are used for partitioning,they are Quad-tree partitions and Horizontal-Vertical (HV)partitions Two innovative techniques are also proposed ,Polygonal blocks of different shapes and Irregular partitions in [8]-[12]. A Quad tree partition provides best rate distortion as compared to fixsize block, polygonal and HV partitions. Temporary images used to form the range blocks are called the domain blocks. The whole process of fractal image encoding involves partitioning the image to form the Range blocks and domain blocks detection of domain blocks and Proper application of affine transforms. Further the choice of domain blocks depends on the type of partition scheme used.[12-14]. The domain pool in fractal encoding is similar to the codebook which is referred to as virtual codebook or domain codebook. The set of transformations selected is applied on each Range block to map on to the domain blocks Fractal Image compression provides high Compression Ratio and good Peak Signal to Noise Ratio. There are wide varieties of approaches being implemented by researchers to fasten the encoding process [15-20]. These approaches are addressed as Speed-up-Techniques for fractal image compression and broadly classified as Domain Classification Based Methods and Feature Vector Based Methods. The field of medical imaging in image processing and its application in the last decades has improved biomedical image processing. Medical images when processed get corrupted due to many aspects by noise and the images need to be filtered using smoothing filters [21-22]. Image enhancement is one of the field in image processing which improves the subjective quality of the image. The pre-processed MRI brain image contains a high intensity salt and pepper noise which appears due to the presence of gray scale variations in the image which is removed by applying suitable filters and performing normalization. Hence the objective of enhancement is de-noising the high frequency components. Adaptive filter was implemented (Tsai et al., 1995) to remove local noisy fluctuations and the outlines of the bone and soft tissues. It suited particularly for texture representation and discrimination. Anisotropic diffusion filter (Amit, 1997) forthe registered images was used. Prewitt edge-finding filter to enhance the image edges robustly. Gaussian filters were also used to enhance the image and make the image gradients stronger. There are as many smoothing filters which improve the edges, sharp corners and enhances the quality of the image The noise in medical images can be introduced due to the machines used for acquiring images. De noising of medical images is a wide research area and many challenging improvements are being conducted in this area to improve the quality of the image.. Bogdan smolka et al. (2002) in this paper a novel approach to the problem of edge preserving smoothing is proposed and evaluated. The new algorithm is based on the combined forward and backward anisotropic diffusion with incorporated time dependent cooling process. This method is able to efficiently remove image noise, while preserving and enhancing its edge Alexei A. Samsonov et al. (2004) in their work designed a new method for filtering MR images with spatially varying noise levels. In the new method, a priori information regarding the image noise level spatial distribution is utilized for the local adjustment of the anisotropic diffusion filter. Gabor filter (Metaxas et al., 2006) was applied to remove the tagging lines and enhance the tag-patterned regions in the image. Julio Martin-Herrero et al. (2007) this paper reviews recent advances in anisotropic diffusion for multi valued images, analyzes their application to hyper spectral images, and proposes a new diffusion method which takes advantage of the recent improvements and conforms to the specificities of hyper spectral remote sensing. Ovidiu Ghita et al. (2010) this paper is concerned with the introduction of a new gradient vector flow (GVF) field formulation that shows increased robustness in the presence of mixed noise and with its evaluation when included in the development of image enhancement algorithms Umamaheswari et al. (2012) presented hybrid method to improve the image quality of Digital Imaging and Communications in Medicine (DICOM) images. The idea of image enhancement technique is to improve the quality of an image for early diagnosis. Then followed by a noise reduction using speckle reduction anisotropic filter introduced by Perona and malik. Many researchers have worked with adaptive, 
modified anisotropic diffusion filters and denoising filters which has significantly improved reduction of noise in medical images [23-37]

\section{FRACTAL THEORY}

This section gives a brief description on the general aspects of fractal image compression and the algorithm for fractal encoding and decoding algorithm is discussed. Fractal Image Compression is based on fractal theory of self similar affine transforms [8-10].

\subsection{Iterated Function Systems and Contractive Affine transforms}

Iterated function systems are a set of collections of contractive transformations denoted by $\mathrm{w}_{\mathrm{i}}$ These transformations maps $\mathrm{R}^{\mathrm{n}}$ to $R^{n}$ itself. $R$ is real valued and indicates the gray level intensities in the two dimensional image.

\subsection{Fractal Image compression}

The Major key issues in Fractal Image coding are partitioning of the image into non overlapping blocks called Range Blocks and Domain Blocks. The Domain Blocks chosen is twice that of the Range Blocks. Selection of suitable Domain Blocks for each Range Block is another key issue This is achieved by applying suitable contractive affine transforms to the domain blocks and the transformed Domain blocks that maps to the Range Blocks forms the fractal code Book. These set of transformed domain Blocks forms a collage to the original image. The Affine transformations that are applied to the image can rotate, stretch, shrink, translate, and skew the input image. A self- affine transformation $\mathrm{w}$ : defined by $\mathrm{R}^{\mathrm{n}}$ to $\mathrm{R}^{\mathrm{n}}$ transformation is of the form $\mathrm{W}(\mathrm{x})=\mathrm{T}(\mathrm{x})+\mathrm{b}$, where $\mathrm{T}$ is a linear transformation on $\mathrm{R}^{\mathrm{n}}$ and $\mathrm{b} \in \mathrm{R}^{\mathrm{n}}$ is a vector

\subsection{Quad-tree Partitioning Technique}

Fractal Image compression though has high compression ratio yet takes a long time for encoding. Many Researchers are working on this aspect, so as to reduce the encoding time. The first step to overcome this drawback is to suitably select the partitioning schemes among the four partitioning schemes. The four schemes are fixed, quad tree, horizontal-vertical and triangular partitioning scheme. In this proposed technique quad-tree partitioning scheme is employed. In Quad-tree Partitioning[8-9] the square image is divided into four equalsized square blocks, and then each block is tested to see if it meets some criteria of homogeneity. If the block meets the criteria, it is not divided any further else if it does not meet the criteria, it is subdivided again into four blocks, and the test criteria are applied to those blocks. This process is repeated iteratively until each block meets the criteria. This technique is illustrated in figure 3.1 shown below

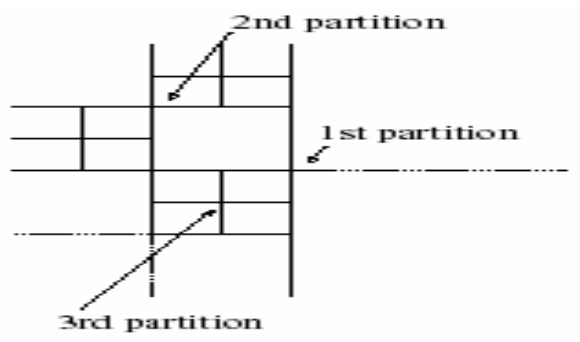

Figure 3.1 quad-tree-partitioning Technique

\subsection{Fractal Image Compression using Quad-tree partitioning technique}

The Input image to be compressed is read and the original image is partitioned into Range blocks according to Quad-tree partitioning method which divides a square image into four equal-sized square blocks, and then each block is tested to see if it meets some criteria of homogeneity. If the block meets the criteria, it is not divided any further. If it does not meet the criteria, it is subdivided again into four blocks, and the test criteria are applied to those blocks.

This process is repeated iteratively until each block meets the criteria. The result may have Range blocks of several different sizes. The criteria met in the present quad-tree method is that, the partitioning method will iteratively divide until it reaches the minimum block dimension of blocks. In this paper minimum Range block sizes of $2 \times 2$ is chosen. Affine transformation is performed on the domain blocks by controlling reflections and rotations indicated by the parameter $c_{\mathrm{k}}, \mathrm{e}_{\mathrm{k}}, \mathrm{f}_{\mathrm{k}}, \mathrm{g}_{\mathrm{k}}$, and $\mathrm{h}_{\mathrm{k}}$ as in equation 1. The Domain Block is affine transformed and is compared with each of the Range block and a closest approximation of the domain block with that of the Range block is found as in equation 1 based on the Root mean squared error calculated between the Range Blocks and the transformed domain Blocks.

$$
\boldsymbol{W}_{k}\left[\begin{array}{c}
x \\
y \\
z
\end{array}\right]=\left[\begin{array}{ccc}
c_{k} & d_{k} & 0 \\
e_{k} & f_{k} & 0 \\
0 & 0 & s_{k}
\end{array}\right]+\left[\begin{array}{l}
g_{k} \\
h_{k} \\
o_{k}
\end{array}\right] \square \square \square \square
$$

The Root Mean square Error $E_{i}$ is calculated by the equation 2

$$
E_{i}=\sqrt{\sum\left[R_{i}(l, k)-D_{i}(l, k)\right]^{2}},
$$

where $R_{i}(l, k)=\mathrm{i}^{\text {th }}$ Range block of size $2 \times 2$ and $D_{i}(l, k)$ is the $\mathrm{i}^{\text {th }}$ Down sampled domain block of size $2 \times 2$ )

The transformed domain block which is found to be the best approximation for the current range block is assigned to that range block and this forms the fractal code book.

The reconstruction process of the original image consists on the applications of the transformations described in the fractal code book iteratively to some initial image until the encoded image is retrieved back. PSNR , CR are calculated for the further analysis of the

\section{DE-NOISING SMOOTHING FILTERS}

Medical Images like MRI of Brain, Ultrasound images, mammograms and CT of Bones are considered in this research paper. As these images are added up with various types of noise during acquisition from instruments and also due to shake of the body while acquisition. These images need to be post processesed after decompression process to remove the noise introduced in the compression and acquisition process. As per the survey there are many de noising smoothing filters and among them anisotropic diffusion filters, Bilateral filters averaging filters, Gaussian filters are being used .This work is an extension of the previous objective which was to analyze the PSNR values obtain from the various smoothing filters and to find its suitability on Medical Images .The current work aims in finding the best filter among anisotropic diffusion filter and Bilateral filter for de noising medical images. Filtering action performed by smoothing filters can be considered as a linear operator that performs convolution operation or sliding of a 
mask over the image. .The Mask or the kernel contains fixed coefficients that overlaps over the image and calculates a weighted sum in the area of overlap Subsections. The different filter action and its convolution kernel are given below.

\subsection{BILATERAL filter}

A bilateral filter is a non-linear, edge-preserving and noisereducing smoothing filter for images. The intensity value at each pixel in an image is replaced by a weighted average of intensity values from nearby pixels. This weight can be based on a Gaussian distribution. And the weights depend not only on Euclidean distance of pixels, but also on the radiometric differences (e.g. range differences, such as color intensity, depth distance, etc.). This preserves sharp edges by systematically looping through each pixel and adjusting weights to the adjacent pixels accordingly.

The bilateral filter is defined through equation 1 and 2

$I^{\text {filtered }}(x)=\frac{1}{W_{p}} \sum_{x_{i} \in \Omega} I\left(x_{i}\right) f_{r}\left(\left\|I\left(x_{i}\right)-I(x)\right\|\right) g_{s}\left(\left\|x_{i}-x\right\|\right)$,

Eqn 1

$$
W_{p}=\sum_{x_{i} \in \Omega} f_{r}\left(\left\|I\left(x_{i}\right)-I(x)\right\|\right) g_{s}\left(\left\|x_{i}-x\right\|\right)
$$

--Eqn2 Ifiltered is the filtered image and I is the original input image to be filtered and $\mathrm{X}$ are the coordinates of the current pixel to be filtered $\Omega$ is the window centered in $X . f_{r}$ is the range kernel for smoothing differences in intensities. This function can be a Gaussian function. $\mathrm{g}_{\mathrm{s}}$ is the spatial kernel for smoothing differences in coordinates. This function can be a Gaussian function. As mentioned the weight $\mathrm{W}_{\mathrm{P}}$ is assigned using the spatial closeness and the intensity difference. Considering a pixel located at $(i, j)$ is required to be de noised in image using its neighboring pixels and one of its neighboring pixels is located at $(\mathrm{k}, 1)$, then, the weight assigned for pixel $(k, l)$ to de noise the pixel $(i, j)$ is given by the equation 3

$$
w(i, j, k, l)=e^{\left(-\frac{(i-k)^{2}+(j-l)^{2}}{2 \sigma_{d}^{2}}-\frac{\|I(i, j)-I(k, l)\|^{2}}{2 \sigma_{r}^{2}}\right)}
$$

eqn3

where $\sigma_{d}$ and $\sigma_{r}$ are smoothing parameters and $\mathrm{I}(\mathrm{i}, \mathrm{j})$ and $\mathrm{I}(\mathrm{k}$, 1) are the intensity of pixels $(\mathrm{i}, \mathrm{j})$ and $(\mathrm{k}, \mathrm{l})$ respectively. After calculating the $I_{D}$ given by equation 4 .

$$
I_{D}(i, j)=\frac{\sum_{k, l} I(k, l) * w(i, j, k, l)}{\sum_{k, l} w(i, j, k, l)}
$$

eqn4

Weights normalize them. Where $I_{D}$ is the de noised intensity of pixel $(i, j)$.

\subsection{Anisotropic diffusion filter}

Anisotropic diffusion filter is also called Perona-Malik Diffusion process.. It is a filter that removes noise without removing important parts of the image. The filters in general follow the scale space approach which is used in edge detection. This was first introduced by perona and Malik[26].Anisotropic diffusion is a process that generates a scale space, where an image generates successively more and more blurred images based on diffusion process. These resulting images in this family are considered to be a convolution operation or combination between the image and a 2D isotropic Gaussian filter, where the width of the filter increases with the parameter. This diffusion process is linear and space-invariant transformation of the original image. Anisotropic diffusion is a generalization of this diffusion process. It produces a family of parameterized images, but each resulting image is a combination between the original image and a filter that depends on the local content of the original image. As a consequence, anisotropic diffusion is a non-linear and space-variant transformation of the original image. They considered the filtered image $I(x, y, t)$ and the original image $\mathrm{I}_{0}(\mathrm{x}, \mathrm{y})$ to be embedded in a family of functions defined by $\mathrm{I}(\mathrm{x}, \mathrm{y}, \mathrm{t})=\mathrm{I}_{0}(\mathrm{x}, \mathrm{y}) * \mathrm{G}(\mathrm{x}, \mathrm{y}, \mathrm{t})$ where $\mathrm{G}(\mathrm{x}, \mathrm{y}, \mathrm{t})$ is a Gaussian kernel with varying scale parameter $t$. In this approach, filtering can be achieved as the iterative solution of the heat diffusion equation where $\mathrm{I}(\mathrm{x}, \mathrm{y}, 0)=\mathrm{I}(\mathrm{x}, \mathrm{y})$ is the initial condition, and $t$ is equivalent to time. Here smoothing is achieved through diffusion process which is stopped at the edges and boundaries

\section{Proposed Fractal Image Compression with denoising filter}

This section discusss issues related to Implementation of the Fractal image compression with De noising filter .The proposed algorithm is done using the following steps and the figure 5.1 shows the general Block diagram for the implementation

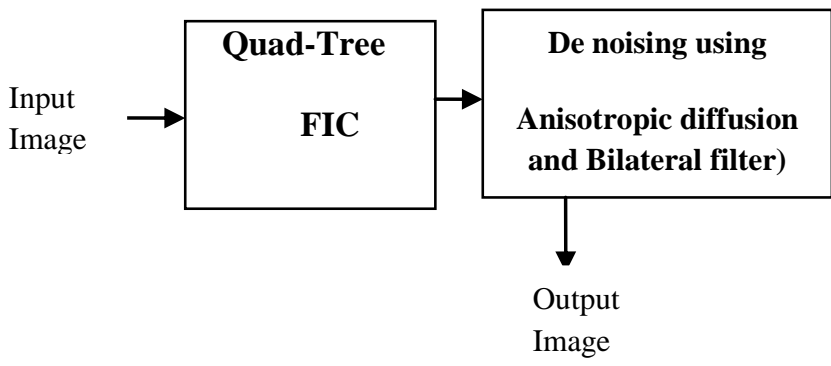

Figure5.1 General Block diagram of Image Processing

- The Medical Images [31-32] to be compressed is read and decomposed using quadtree partitioning technique.

- Affine transforms are applied to the domain blocks so as to suitably match the Range blocks

- The Transformed Domain Block which is found to be the best approximation to the Range block is stored in the fractal code book

- The Fractal Compressed image is then De noised using smoothing filters like median, mean, anisotropic diffusion filter and Gaussian filter to remove noise

- $\quad$ PSNR for both FIC with and without Filtering is noted and compared for further analysis.

\section{OBJECTIVE MEASURES}

The images are analyzed based on subjective and objective measures .Subjective measures are based on decisions made by individuals and differs from person to person. Objective measures are based on statistical parameters extracted from images. The objective measures which are calculated from the parameters obtained from the images are Peak Signal to Noise Ratio, Compression Ratio,

\subsection{Peak signal to Noise Ratio}

Signal to Noise Ratio (SNR) is an objective measure and estimates the quality of the image .If $f(i, j)$ is the original image and $\mathrm{F}(\mathrm{I}, \mathrm{J})$ is the reconstructed image then Peak signal to Noise ratio is computed using equation 1 
PSNR $=20 \log _{10}\left[\frac{\mathrm{N}}{\mathrm{RMSE}}\right] \mathrm{dB}$

Where $\mathrm{N}$ is the number of Gray level values of the image

\subsection{Compression Ratio}

Compression ratio is defined as the ratio between the number of bits required to represent the ouput image and the number of bits required to represent the input image.

\section{RESULTS AND DISCUSSIONS}

\subsection{Methodology}

A Bulk data of Medical images each of hundred images of Magnetic Resonance Images [33-34] of brain (T1 and T2 weighted) CT Of bone, ultrasound images[US] of uterus, Mammograms of size $256 \times 256$ and 8 bit gray scale were collected from JSS Hospital and Vikram Hospital Mysor for the current research work.. Fractal image compression using quad-tree partitioning technique with and without filters were applied on these images. A minimum Range Block size of $2 \times 2$ was considered. Peak Signal to Noise Ratio (PSNR) were computed for the two type of filters and the results are tabulated as shown in Table 7.1 below

\subsection{Results}

The Mat lab Simulated Results shown in the table 7.1explains that fractal image compression with filters shows a better PSNR value than when fractal Image compression is post processed without Filter .The quality of image is also being preserved. Which is shown in figures 7.1? As PSNR is a significant factor in determining the objective analysis of the image, it is seen that FIC works better after processing using Bilateral filters than when compared to anisotropic diffusion filter. The PSNR value is improved to $94.4 \mathrm{~dB}$ from $30.4 \mathrm{db}$.

Table 7.1 a Comparison of PSNR values for Bilateral and anisotropic filters for MR images

\begin{tabular}{|l|l|l|}
\hline \multirow{2}{*}{ Filter Type } & \multicolumn{2}{|l|}{ MR Image } \\
\cline { 2 - 3 } & With filter & Without Filter \\
\hline Bilateral Filter & 93.6026 & 29.0 \\
\hline Anisotropic Filter & 85.73 & 29.0 \\
\hline
\end{tabular}

Table 7.1b Comparison of PSNR values for Bilateral and anisotropic filters for Mammograms images

\begin{tabular}{|l|l|l|}
\hline \multirow{2}{*}{ Filter Type } & \multicolumn{2}{|l|}{ Mammograms } \\
\cline { 2 - 3 } & With filter & Without Filter \\
\hline Bilateral Filter & 94.4726 & 35.0 \\
\hline Anisotropic Filter & 87.43 & 35.0 \\
\hline
\end{tabular}

Table 7.1c Comparison of PSNR values for Bilateral and anisotropic filters for CT images

\begin{tabular}{|l|l|l|}
\hline \multirow{2}{*}{ Filter Type } & \multicolumn{2}{|l|}{ CT image } \\
\cline { 2 - 3 } & With filter & Without Filter \\
\hline Bilateral Filter & 88.2063 & 30.8 \\
\hline Anisotropic Filter & 80.47 & 30.8 \\
\hline
\end{tabular}

Table 7.1 d Comparison of PSNR values for Bilateral and anisotropic filters for ULTRASOUND images

\begin{tabular}{|l|l|l|}
\hline \multirow{2}{*}{ Filter Type } & \multicolumn{2}{|l|}{ Ultrasound Image } \\
\cline { 2 - 3 } & With filter & Without Filter \\
\hline Bilateral Filter & 89.2699 & 30.4 \\
\hline Anisotropic Filter & 84.04 & 30.4 \\
\hline
\end{tabular}

The simulated Results of the simulation are shown in figure 7.1, figure 7.2 figure 7.3 and figure 7.4 for both with and without filters.

QuadTree

Original MR Image

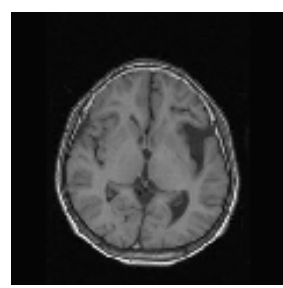

Threshold: 0.10

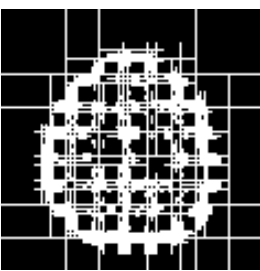

Quadtree Decoded Image

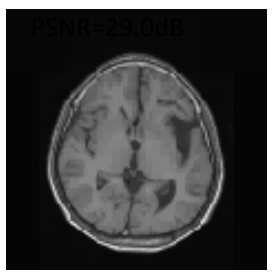

Figure: 7.1a Matlab simulated Result for MR Image using Quad-Tree FIC without Filter
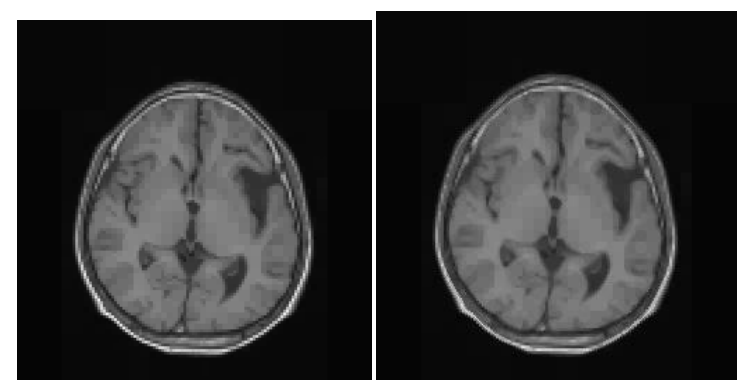

Figure:7.1b Matlab simulated Result for Post Processed Quad-Tree FIC MR image using Anisotroic diffusion filter and Biltaeral filter

Threshold: 0.10
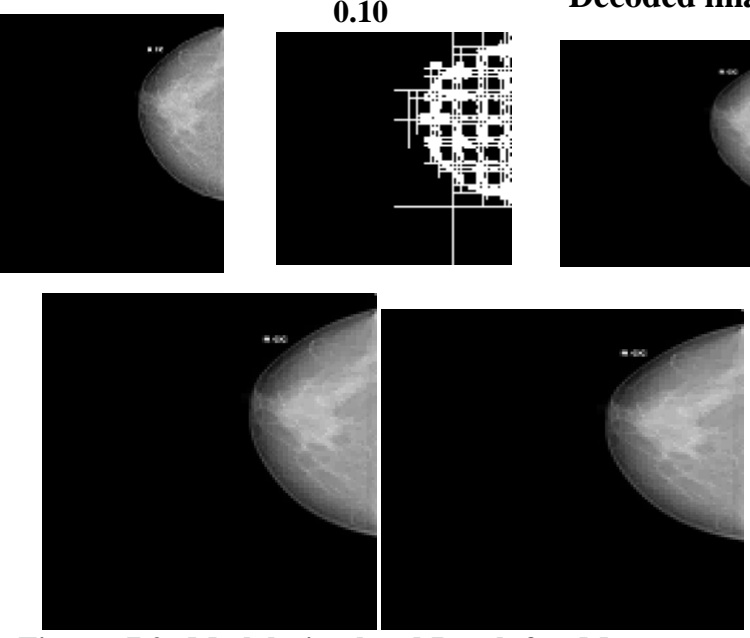

Figure: 7.2a Matlab simulated Result for Mammogram using Quad-Tree FIC without FIlter

Figure: 7.2b Matlab simulated Result for Post Processed Quad-Tree FIC Mammogram image using and Anisotroic diffusion filter and Bilteral 


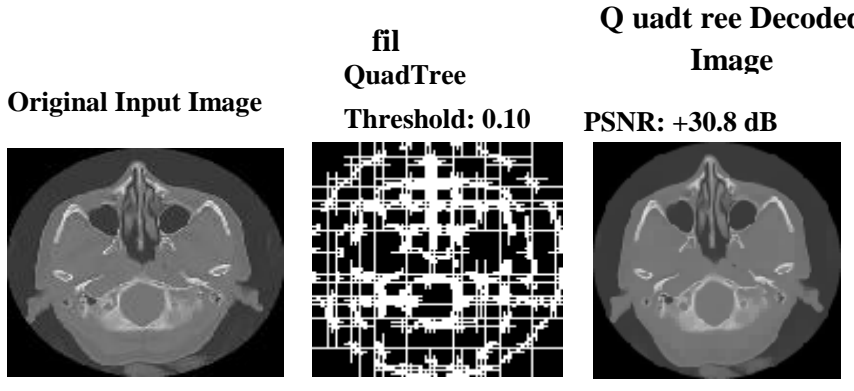

Figure: 7.3a Matlab simulated Result for CT Image using Quad-Tree FIC without Filter
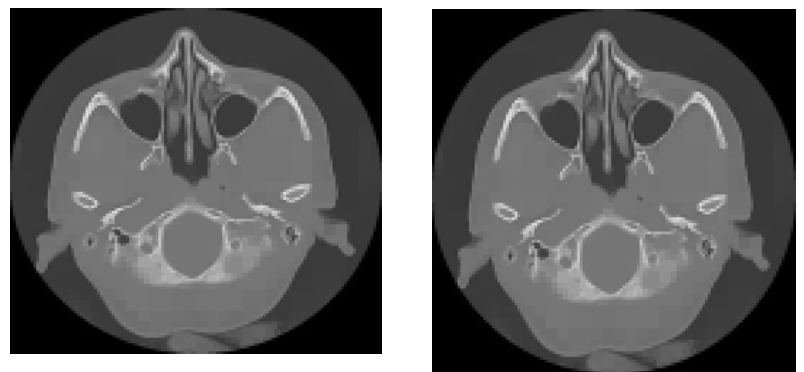

Figure: 7.3b Matlab simulated Result for Post Processed Quad-Tree

FIC CT image using anisotrpic difussion filter and Bilteral

$$
\text { QuadTree Quadtree Deooded Image }
$$
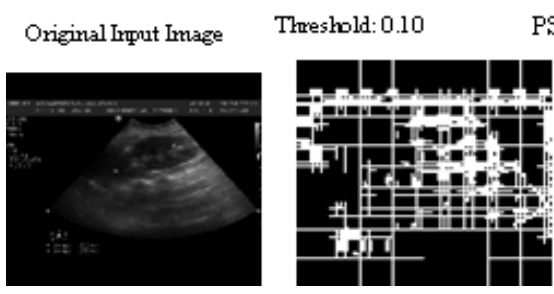

PSNR: $+30.4 \mathrm{~dB}$

filter

Figure 7.4a Matlab simulated Result for Ultrasound Image using Quad-Tree FIC without Filter
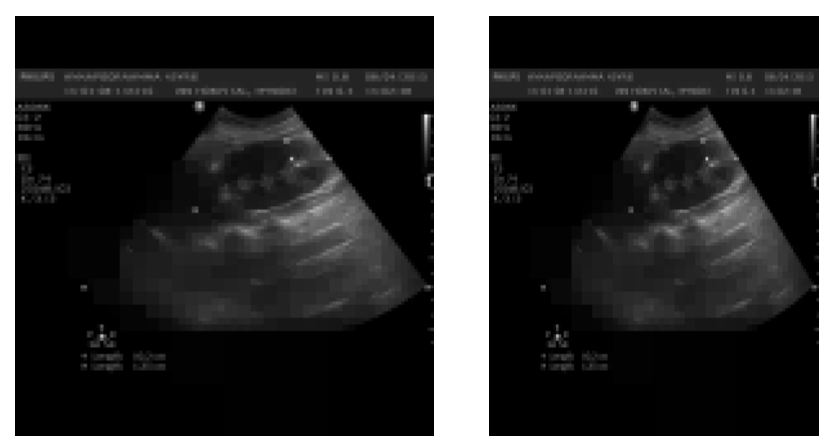

Figure :7.4b Matlab simulated Result for Post Processed Quad-Tree FIC Ultrasound Image using and Anisotroic diffusion filter AND Bilteral filter

\section{CONCLUSIONS}

The simulated results obtained shows that there is improvement in PSNR value to $93.6 \mathrm{Db}, 88.2 \mathrm{Db}, 94.4 \mathrm{Db}, 89.2 \mathrm{Db}$ is obtained for different medical images using Bilateral filter. It is also observed from the graphs shown in figure 8.1 it is observed that bilateral filters are better suited for medical images than anisotropic diffusion filter as it provides better PSNR value. Hence for post processed fractal compressed image bilateral filter is best suited for noise removal. Also the PSNR value is more for Mammograms than when compared to other imaging modalities; Hence FIC is also best suited for Mammograms. The results can also be modeled and implemented using FPGA.

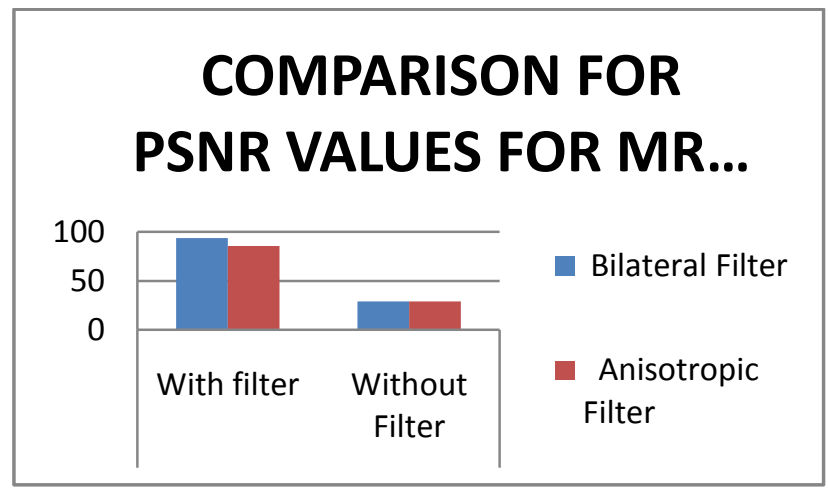

Figure 81a comparison of PSNR values for MR Images

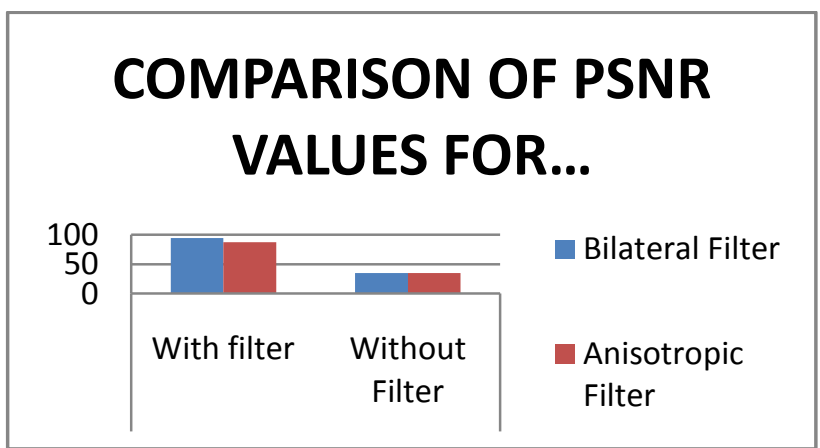

Figure 8.1b comparison of PSNR values for Mammogram Images

\section{COMPARISION OF PSNR VALUES FOR ULTRASOUND...}

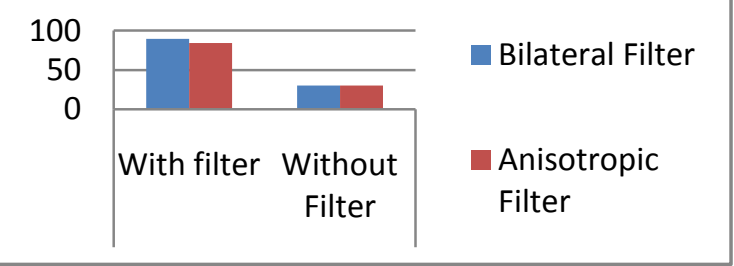

Figure 8.1c comparison of PSNR values for ultrasound Images 


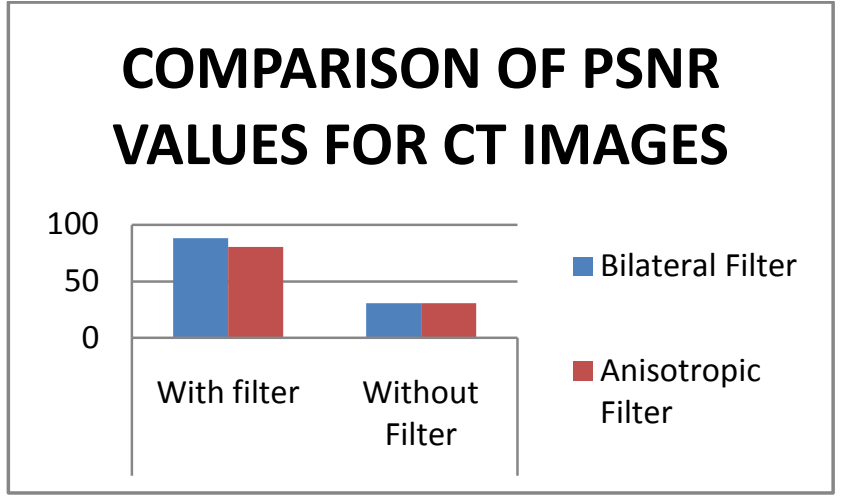

Figure 8.1a comparison of PSNR values for CT Images

\section{REFERENCES}

[1] S.Bhavani - "A Survey On Coding Algorithms In Medical Image Compression (IJCSE) International Journal on Computer Science and Engineering Vol. 02, No. 05, 2010, 14

[2] Fractal Compression of Medical Images -A Master Thesis-Wojciech Walczak

[3] M.J. Zkoski, T.Boult and T. Iyriboz, "A Novel approach to medical image Compression" , Intl.J. Bioinformatics Research and Applications, Vol2, No.1, 2006

[4] Miroslav Galabov-Fractal Image compression' "International Conference on Computer Systems and Technologies - CompSysTech’2003

[5] N.A.koli and M.S.Ali-“A survey on Fractal Compression Key issues” International technology Journal -2008

[6] [6] John Leis-"Fractal coding Lectue Notes"-2006

[7] M. Barnsley, Fractals Everywhere. New York: Academic,(1988).

[8] A.E. Jacquin, "Image coding based on a fractal theory of iterated contractive image transformation", IEEE Trans. On Image Processing, 1(1): (1992)

[9] Arnaud E. Jacquin, (1993) "Fractal image coding", Proceedings of IEEE VOL.81, pp. 1451-1465

[10] Y. Fisher, Fractal Image Compression: Theory and Application. New York: Springer-Verlag, (1994).

[11] R. Distasi, M. Nappi and D. Riccio, "A range/domain approximation error- based approach for fractal image compression", IEEE Trans. Image processing, vol. 15, no. 1, pp. 89-97, Jan. 2006.

[12] Vijayshri Chaurasia et al. / International Journal of Engineering Science and Technology Vol.2 (2), 2010, 104-108

[13] Y.Fisher,E.W.Jacobs and R.D.Boss,'Fractal Image compression using Iterated transforms in image and text compression" J A storer,Ed Boston MA Kluwer ,pp 3561(1992)

[14] .L Thomas and F.Deravi “ Region based fractal image compression using heuristic speech "IEEE transaction on Image processing 4(6) 832-836 (1995)

[15] Tanimoto, H. Ohyama and T. Kimoto, “ A new fractal image coding scheme employing blocks of variable
shapes",IEEE Int. Conf. Image Processing, Lausanne Switzerland,1: 137-140(1996).

[16] Ruhl, H. Hartenstein and D. Saupe, "Adaptive partitioning for fractal image compression", IEEE Int. Conf. Image Processing, Santa Barbara, CA, 2: 310313(1997).

[17] Y. Chakrapani, K. Rajan. "Implementation of fractal image compression employing artificial neural networks ISSN 1 746-7233, England, UK World Journal of Modeling and Simulation Vol. 4 (2008) No. 4, pp. $287-$ 295

[18] Dr. Fakhiraldeen H. Ali Quad-tree Fractal Image Compression University of Mosul

[19] M.S.Soyjaudah and I.Jahmeerbacus "Fractal image compression using quad-tree partitioning" International Journal of Electrical Engineering Education 39/1

[20] Pamela,, Gray,R.M.olshenA.(1994b)"Evaluating Quality of Compressed Medical Images: SNR, Subjective Rating and Diagnostic Accuracy', Proc. of the IEEE, Vol. 82, pp. 920-931.

[21] S.Vijaykumar African Journal of Mathematics and Computer Science Research ,Vol69,pp-179-182-oct2013 ISSN-2006-973 Hybrid filters for medical image reconstruction

[22] Gajanand Gupta , International Journal of Soft Computing and Engineering (IJSCE)ISSN: 2231-2307, Volume-1, Issue-5, November 2011, "Algorithm for Image Processing Using Improved Median Filter and Comparison of Mean, Median and Improved Median Filter

[23] Asish verma "et.al" International Journal of Computer applications (0975 - 8887) Volume 1 - No. Comparative Analysis in Medical Imaging"

[24] Raka Kundu* and Amlan Chakrabarti** Research frontDenoising image filters for biomedical image processing

[25] Pietro perona and Jitendra Malik "Scale space and edge detection using Anisotropic diffusion "IEEE transactions on Pattern analysis and Machine Intelligence, vol 12,no 7 1990.

[26] Shokhan Mahmoud Hama1 and Muzhir Shaban Al-Ani2 University of Al-Anbar, Collage of Computer, Anbar, Iraq International Journal of Advances in Engineering \& Technology, july 2013. Cijaet issn: 22311963 "Medical image enhancement based on an efficient approach for adaptive anisotropic diffusion

[27] Garima Goyal1, Ajay Kumar Bansal2, Manish Singhal3 International Journal of Scientific and Research Publications, Volume 3, Issue 1, January 20131 ISSN 2250-3153"Review paper on various filtering techniques and future scope to apply these on TE M images"

[28] Mohamed Saleh Abuazoum "Efficient analysis of medical image de-noising for mri and ultrasound images"a Dissertation

[29] Sannella, M. J. 1994 Constraint Satisfaction and Debugging for Interactive User Interfaces. Doctoral Thesis. UMI Order Number: UMI Order No. GAX9509398., University of Washington. 
[30] Forman, G. 2003. An extensive empirical study of feature selection metrics for text classification. J. Mach. Learn. Res. 3 (Mar. 2003), 1289-1305.

[31] Al-Nauimy, W "Comparative Study between Different Denoising Filters for Speckle Noise Reduction in Ultrasonic BMode Images, IEEE, July, 2012.

[32] Asoke Nath, Image Denoising Algorithms: A Comparative study of Different Filtration approaches used in image restoration, IEEE, International Conference on Communication Systems and Network Technologies, 2013.

[33] Charu Khare, Kapil Kumar Nagwanshi , Image Restoration Technique with Non Linear Filter , International Journal of Advanced Science and Technology Vol. 39, February, 2012 .

[34] Jianjun Zhang, An efficient median filter based method for removing random valued impulse noise, Science Direct, Digital Signal Processing 20 (2010).
[35] Laxmi Laxman, V Kamalaveni, K A Narayanankutty, Comparative Study On Image Restoration Techniques Using The Partial Differential Equation And Filters, International Journal of Engineering Research \& Technology (IJERT),Vol. 2 Issue 7, July 2013, pp: 55-59.

[36] Rohit Verma, Dr. Jahid Ali, AComparative Study of Various Types of Image Noise and Efficient Noise Removal Techniques, International Journal of Advanced Research in Computer Science and Software Engineering, Volume 3, Issue 10, October 2013, pp: 617-622.

[37] Shabnam Sultana, M.Varun Kumar, N.Asha, Comparison of Image Restoration and DenoisingTechniques , International Journal of Advanced Research in Computer Science and Software Engineering, Volume 3, Issue 11, November 2013, pp: 337-341. 